\title{
Wearable Technology and How This Can Be Implemented into Clinical Practice
}

\author{
Justin Greiwe ${ }^{1,2}$. Sharmilee M. Nyenhuis ${ }^{3,4}$ \\ Published online: 6 June 2020 \\ (C) Springer Science+Business Media, LLC, part of Springer Nature 2020
}

\begin{abstract}
Purpose of Review Our day-to-day life is saturated with health data that was previously out of reach. Over the last decade, new devices and fitness technology companies are attempting to tap into this data, uncovering a treasure trove of useful information that, when applied correctly, has the potential to revolutionize the way we approach healthcare and chronic conditions like asthma, especially in the wake of the COVID-19 pandemic.

Recent Findings By harnessing exciting developments in personalization, digitization, wellness, and patient engagement, care providers can improve health outcomes for our patients in a way we have never been able to do in the past. While new technologies to capture individual health metrics are everywhere, how can we use this information to make a real difference in our patients' lives? Navigating the complicated landscape of personal wearable devices, asthma inhaler sensors, and exercise apps can be daunting to even the most tech savvy physician.

Summary This manuscript will give you the tools necessary to make lasting changes in your patients' lives by exposing them to a world of usable, affordable, and relatable health technology that resonates with their personal fitness and wellness goals. These tools will be even more important post-COVID-19, as the landscape of clinical outpatient care changes from mainly in-person visits to a greater reliance on telemedicine and remote monitoring.
\end{abstract}

Keywords Telemedicine $\cdot$ Wearable devices $\cdot$ Personalized healthcare devices $\cdot$ Remote monitoring $\cdot$ COVID-19 $\cdot$ Connected health

\section{Introduction}

As our world has become upended with the arrival of COVID19 , the way in which healthcare will be provided in the future

This article is part of the Topical Collection on Telemedicine and Technology

Justin Greiwe

jcgreiwe@gmail.com

1 Bernstein Allergy Group, Inc, 8444 Winton Road, Cincinnati, OH 45231, USA

2 Division of Immunology/Allergy Section, Department of Internal Medicine, The University of Cincinnati College of Medicine, Cincinnati, OH, USA

3 Division of Pulmonary, Critical Care, Sleep and Allergy, Department of Medicine, University of Illinois at Chicago, Chicago, IL, USA

4 Center for Dissemination and Implementation Science, Department of Medicine, University of Illinois at Chicago, Chicago, IL, USA is likely to change drastically. This pandemic is a big wake-up call for limiting in-person visits, especially during the flu season that put vulnerable patients at undo risk coming into the office for routine care. We have had the technology to perform telemedicine visits for years, but patients' hesitancy and poor insurance reimbursement have always been barriers for widespread acceptance. In the wake of the COVID-19 pandemic, most primary care and specialty offices transitioned to a telemedicine-focused approach within a matter of days to weeks. In response, the Centers for Medicare \& Medicaid Services (CMS) relaxed many requirements for Medicare patients and the states followed suit for Medicaid patients. Insurance companies also loosened restrictions giving healthcare providers increased flexibility to provide care for their patients from the comfort and safety of their own homes. 2020 will be the ultimate testing ground for various telehealth services and remote monitoring applications, and it is the hope of the authors that recent changes in access and reimbursement will remain in place permanently. To meet the needs of this growing market, new technologies including wearable 
devices will play an important role in providing real-time data to objectively monitor patients at home. These devices will be especially important for improving the health and control of chronically ill patients and for those with conditions like asthma, COPD, diabetes, and cardiovascular disease. The focus of this paper revolves around wearable devices for asthma, but can be applied for any chronic condition. If these home devices can provide usable data in a reliable, cheap, and easy-touse format, then the need for in-person visits in the future will be drastically reduced.

\section{Connected Health}

Innovations in personalized health technology provide a unique opportunity to develop connected healthcare models that are built around the patients' individual needs. The term connected health describes the use of a variety of technologies to inform and aid healthcare delivery in a data-driven manner with the individual at the center. ${ }^{7}$ It covers a broad domain, including digital, mobile health, and telehealth, and ensures all stakeholders are connected with data that is accurate and timely [1]. An integrative connected health model can also offer social support through online forums or networks where users are able to interact with peers with similar healthcare conditions or goals helping to further enhance patient experience and improve adherence.

Commercial weight loss programs like WW (formerly Weight Watchers) was one of the first large-scale programs to take advantage of social support networks to motivate their clients and make them accountable to other people. A 2005 systematic review of commercial and proprietary weight loss programs' efficacy concluded that WW was the only program with demonstrated efficacy in achieving modest weight loss based on results from 3 randomized controlled trials (RCTs), and their unique social approach likely contributed to its success [2]. A recently published systematic review found relatively strong evidence that greater social support, self-efficacy, and selfmotivation can predict higher adherence to home-based therapies [3]. There is enough data to suggest that social support networks improve adherence to fitness, weight loss, and home-based therapies that healthcare providers should be actively curating a wider support network for their patients [4-6]. Wearable devices and associated apps take social connectivity to the next level and provided unparalleled access to support networks that have the potential to further encourage adherence to fitness and health regimens. Promising results have already been reported with the use of apps to improve adherence and outcomes in various healthcare scenarios including weight loss, smoking cessation, diabetic management, and home exercise programs [7-11].
The First Wave of Wearable Devices: Activity Trackers

The concepts of wellness and mindfulness have become an increasing part of the public health discussion as there seems to be a growing appreciation for a more holistic approach to fitness and well-being. With the advent of more advanced wearable technology like fitness trackers and smartwatches starting in 2014, consumers are no longer content with just tracking their weight loss goals or step count. Personalized healthcare devices arm users with snapshots of their activity and recovery data and allow them to track advanced performance metrics including step tracking, heart rate, body fat percentage, sleep quality, stress levels, menstrual cycle, and fertility windows. As this data becomes more accessible, consumers are discovering that overall health is the sum of all the small choices you make each day: what you eat, how much you move, how much you sleep, etc. Having fitness and weight loss goals is an important first step, but converting that enthusiasm to actual gym time and change in habits has always been the rate limiting step. That is where innovative fitness startups and fitness technology companies are focusing their attention: getting people excited about working out, offering unparalleled convenience, and keeping members engaged long-term by utilizing the latest in technology and social media. Since 2014, the use of commercial wearable devices has doubled. Currently, $19 \%$ of Americans use a wearable fitness tracker (e.g., Fitbit, Apple Watch) with sales projected to double by 2022, becoming a $\$ 27$ billion+ market $[12,13]$.

\section{Wearable Devices that Promote Health and Fitness}

As more and more patients use wearable devices and smartphone apps to track their personal health data, how can this information be used in a meaningful way? Fitness technology companies like Fitbit and Apple Health are leveraging insights from over 10 years of activity, exercise, and sleep data to better inform future applications and provide behavior change techniques and insights to their users. Apple recently announced that customers in the USA can enroll in three landmark health studies: the Apple Women's Health Study, the Apple Heart and Movement Study, and the Apple Hearing Study. Conducted in partnership with leading academic and research institutions like the World Health Organization, these multi-year longitudinal studies are leveraging the ubiquity of Apple products to provide a treasure trove of user data to inform potential medical discoveries that could improve healthcare for all [14].

Wearable devices themselves can provide crude feedback like reminders to move or stand which prompts users to take more steps per hour or stand more frequently. For additional 
charges, some devices can provide more detailed personal wellness reports, offering actionable guidance and coaching to help users achieve their health and fitness goals. Some companies like Fitbit are taking it a step further and offering personal, one-on-one coaching services for weight loss and diabetes control providing users with a personalized plans and direct support from their team of certified health and wellness coaches. A small sampling of highly rated fitness and nutrition apps are included in Table 1.

The ultimate goal with these devices and apps is to provide real-time feedback to patients so that they actually see data that illustrates how destructive behaviors impact them physically, providing additional motivation to more proactively manage their health. The more the patient understands about their personal health, the more empowered they will be to make meaningful behavioral changes. Healthcare providers need to be able to access and interpret personalized health data, as well as distill it into usable teaching points. In the past, physicians often took an authoritarian approach to health and fitness education which did little to encourage active participation by the patient. By employing a patient-centered approach utilizing data gleaned from wearable devices, healthcare providers have an opportunity to work in concert with patients to impart measurable skills and behaviors in a real and tangible way with the ability to track this progress over time. When patients are included in a collaborative and patient-centered discussion about their health and well-being, compliance and satisfaction rates improve, leading to a more effective doctor-patient relationship [15-17]. It is important to note that these devices are still in their infancy, and the accuracy and validity of the data still need to be strengthened in order to provide the best medical advice.

\section{Wearable Devices in Asthma}

Most of the wearable fitness device research to date has focused in diseases associated with low levels of physical activity, namely cardiovascular disease, diabetes, and cancer. Few have focused on asthma and of the studies conducted in asthma populations, all have honed in on the use of Fitbit-derived sleep measures (i.e., sleep quality). Castner et al. used the Fitbit Charge ${ }^{\mathrm{TM}}$ to validate sleep measures in women with asthma [18••]. The sleep measures collected by the Fitbit were compared with Actigraph GT3X +, a well-known and validated device to collect sleep measures [19, 20]. The Fitbit device tended to overestimate sleep efficiency and underestimate wake counts compared with actigraphy. Two additional studies were conducted in pediatric populations and focused on correlating Fitbit-derived sleep measures to patient-reported outcomes such as asthma control and asthma impact [21, 22]. Bian and colleagues assessed the association between self-reported asthma impact and Fitbit-derived sleep quality (the ratio of minutes asleep to minutes in bed) and physical activity measures (daily minutes of moderate and vigorous activity) in adolescents with asthma [21]. Fitbit-derived sleep quality was moderately correlated with Patient-Reported Outcomes Measurement Information System (PROMIS) sleep disturbance score $(r=-0.31, P=0.01)$ and had a weak but significant correlation with the PROMIS pediatric asthma impact score (average $r=-0.18, P=0.02$ ). Fitbit-derived physical activity levels were not associated with PROMIS pediatric asthma impact $(r=0.04, P=0.62)$. These findings suggest that measurement of sleep quality, using the Fitbit device, may help develop personalized asthma management strategies for children and their caregivers in real time. The final study measured several mobile metrics of asthma including physical activity and sleep using Fitbits, forced expiratory volume in $1 \mathrm{~s}$ and peak expiratory flow, indoor air quality using Foobot (https://foobot.io/), and a mobile app that collected information on asthma control (symptoms, physical limitations due to asthma, nighttime awakenings, and medication intake) [22]. These metrics were used together to digitally phenotype children with asthma and provide a better measure of the patient's asthma control to their clinician when compared with the Asthma Control Test scores taken infrequently during clinic visits. Additional work using this ecological metric of asthma control is needed and may be used in the future to generate insights on the relationship between a patient's asthma symptoms and triggers across different seasons [23].

Acoustic monitoring is another type of wearable devices that has been studied in asthma. Breathing sound measured by microphones over human skin can detect breathing patterns (respiratory rate, flow rate, tidal volume) and symptoms that may be due to asthma (wheeze, cough). Moreover, chest movement signals can be acquired using an accelerometer or belt-shaped device [24, 25]. Boner et al. measured the nocturnal wheeze in children with asthma using an acoustic respiratory monitor. They found that among children with apparently well-controlled asthma, $57 \%$ had considerable amounts of night wheezing that was unrelated to conventional measures of lung function [26]. The use of these continual wearable monitoring systems is still a developing field that needs further study on its clinical impact.

\section{Use of Electronic Monitoring Devices in Asthma}

Electronic monitoring devices (EMDs) in asthma are increasingly more common since they provide objective data and are not biased by patient self-report. The majority of the currently available EMDs focus on addressing medication adherence, with a limited few focusing on sleep monitoring and identifying asthma triggers. The devices measuring adherence use a 
Table 1 Top rated fitness and nutrition apps

\begin{tabular}{|c|c|c|c|}
\hline App & Supporting devices & Cost structure & Details \\
\hline \multicolumn{4}{|c|}{$\begin{array}{l}\text { For all-around healthy } \\
\text { lifestyle }\end{array}$} \\
\hline 8 fit & iOS, Android & $\begin{array}{l}\text { Free; } \$ 59.99 \text { per year; } \$ 44.99 \\
\text { per half-year; } \$ 19.99 \text { per } \\
\text { month for meal plans and } \\
\text { more workouts }\end{array}$ & $\begin{array}{l}\text { On-demand workouts and meal planning for people } \\
\text { who like a lot of guidance, suggestions, reminders, } \\
\text { and instructions. Provides realistic assessment of } \\
\text { how hard or easy it will be to reach your health and } \\
\text { fitness goals. }\end{array}$ \\
\hline
\end{tabular}

Yoga

Asana Rebel

iOS, Android

Yoga Wake Up

Alo Moves

YogaGlo

On-demand workouts

Peloton Digital

Fitbit Coach

Nike Training Club

Sworkit

Aaptiv

iOS, Android

\$15 per month

$\$ 10$ per month

\$20 per month

\$18 per month Premium

iOS, Android Premium

$\$ 10$ per month

iOS, Android
\$39.99 for 3 months; \$58.99 Yoga-inspired fitness that has guided yoga flows for 12 months

\$20 per month

Free; $\$ 9.99$ per month or $\$ 79.99$ per year for

Free; $\$ 14.99$ per month or $\$ 119.99$ per year for 
Table 1 (continued)

\begin{tabular}{|c|c|c|c|}
\hline App & Supporting devices & Cost structure & Details \\
\hline Charity Miles & iOS, Android & Free & $\begin{array}{l}\text { Motivates by donating money to charity for every mile } \\
\text { users run, walk, or bicycle. Supports a variety of } \\
\text { nonprofit organizations, such as ASPCA, Habitat for } \\
\text { Humanity, St. Jude Children's Hospital, UNICEF, } \\
\text { Save the Children, and the Wounded Warrior } \\
\text { Project. }\end{array}$ \\
\hline
\end{tabular}

Nutrition-Tracking

MyFitnessPal

iOS, Android

Free; $\$ 9.99$ per month or $\$ 49.99$ per year for Premium

Noom
MyPlate
WW Digital (formally
WeightWatchers)

WeightWatchers)

Mindfulness and meditation

Calm

iOS, Android $\$ 14.99$ per month or $\$ 69.99$
per year

$\$ 59$ per month, \$199 per year (additional pricing options available)

iOS, Android, Web Free; $\$ 9.99$ per month, $\$ 30$ for six months, or $\$ 44.99$ per year for Gold

iOS, Android, Web \$23.99 per month or \$219.99 per year

Headspace

iOS, Android

$\$ 12.99$ per month or $\$ 69.99$ per year

The Mindfulness App

iOS, Android
Calorie counting app that also figures out how many calories users should eat to gain, lose, or maintain weight. Consolidates everything users eat and drink, plus all their activities to calculate calories consumed against calories burned. It also has tools for tracking weight and other body changes over time. It has the largest database of foods and is the best food logging app on the market.

Complete weight loss program with daily interactive content, a personal goal coach, and plenty of content to help users master the psychology involved with changing their relationships to food.

All-in-one fitness app, combining calorie counting, weight management, recipes, and workouts. Users look up and record everything they eat and can see a nutritional analysis of macronutrient intake for free (requires paid subscription in MyFitnessPal).

Highly supportive community and app that focuses more on personalization and overall health rather than weight loss alone. Users do not have to attend meetings or meet with a counselor (although this is an option for an added fee). Monitors food intake with SmartPoints rather than counting calories or fat.

Guided mindfulness meditation that initially offers a " 7 Days of Calm" course which introduces users to mindfulness meditation, as well as some guided and unguided meditation sessions. Paid users have access to more multi-day courses, a "Daily Calm" course, and many, many more guided meditations.

Guided meditation for health issues, emotions, challenges, and productivity. There are even "SOS" sessions for some of the more stressful times in life. Recently added free content called Weathering the Storm for those struggling with the emotional effects of COVID-19.

Starts with a 5-day introduction to mindfulness along with guided meditations to get you familiar with meditating. With subscription choose guided or silent meditation sessions for challenges, travel, sleep, relationships, stress relief, emotions, body awareness, focus, and relationships.

*The authors are aware of the transient nature of fitness, nutrition, and lifestyle apps and understand that the recommendations provided at the time of this publication might not be available in the future

variety of approaches from recording audio during inhaler use, using time and date to detect medication use and capturing spirometry measures. These devices are paired with mobile applications (Apps) on smartphones which can provide the 


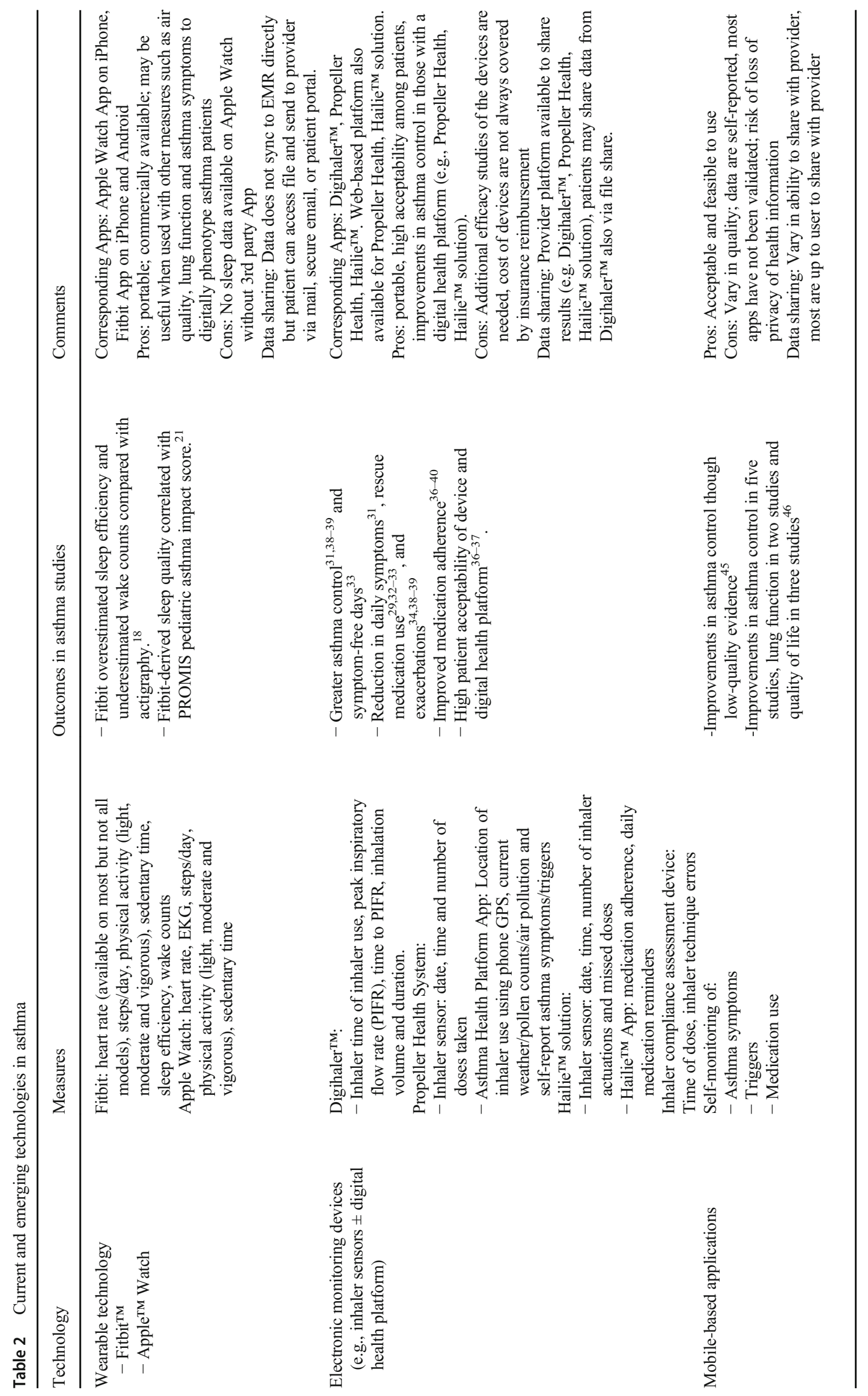


patient with audiovisual reminders for taking medications, correlate medication use with environmental exposures (i.e., weather, ozone, pollution) using smartphone GPS coordinates and feedback on proper inhaler use. A summary of some of the current and emerging technologies in the asthma space is provided in Table 2.

Teva's Digihaler ${ }^{\mathrm{TM}}$ has been FDA approved for use with ProAir® (albuterol sulfate $117 \mathrm{mcg}$ ), AirDuo ${ }^{\circledR}$ (fluticasone propionate and salmeterol), and ArmonAir (fluticasone propionate) inhalers. The Digihaler ${ }^{\mathrm{TM}}$ contains built-in sensors that records inhalation data with timestamps. The inhalation data includes parameters that are important for assessing inhaler technique including peak inspiratory flow rate and inhalation duration [27]. The device is connected via Bluetooth ${ }^{\circledR}$ technology to a companion App that provides information on inhaler use to the patient. While to date, the efficacy of Digihalers has not been evaluated; one pilot study has shown its ability to predict exacerbations [28]. Adults with exacerbation-prone asthma $(n=360)$ used the ProAir ${ }^{\circledR}$ Digihaler $^{\mathrm{TM}}$ for 12 weeks. The Digihaler ${ }^{\mathrm{TM}}$ was able to predict asthma exacerbations (defined as need for oral corticosteroids) with an area under the receiver operating characteristic curve of 0.75 . The most predictive factor was the average number of albuterol inhalations per day during a period of 5 days before an exacerbation. Further developments of the model in larger populations are needed to determine the efficacy of the Digihaler ${ }^{\mathrm{TM}}$ to predict exacerbations.

Propeller Health has developed a system which includes a sensor that can attach to both rescue and controller inhalers and an Asthma Health Platform [29]. The inhaler sensor collects date and time of inhaler use and number of puffs taken. This data is transferred via Bluetooth ${ }^{\circledR}$ technology to the Asthma Health Platform on a mobile device and can be matched with GPS coordinates obtained from the mobile device. The Asthma Health Platform can also be used to record asthma symptoms and triggers which integrates with other data streams such as weather, pollen counts, and pollution. The Propeller Health System is one of the most studied to date. A 4-month pilot study conducted in 30 individuals showed when the device and app were used along with weekly feedback, participants had an improvement in asthma control and a decline in day-to-day asthma symptoms [30]. The effectiveness of Propeller Health System on rescue inhaler has also been examined [31・•]. In a study of 495 patients, all patients received the Propeller Health System but only 250 received sensor-based feedback. The authors found a decrease in mean daily rescue medication use in those receiving sensor-based feedback $(0.19$ vs. 0.25 in control arm) which was more pronounced in patients with uncontrolled asthma at baseline. These findings were confirmed in another study of 120 participants with a decrease in rescue medication use by $39 \%$ and an increase in symptom-free days by $12 \%$ [32]. Propeller Health has also developed a provider-facing web interface (portal) that allows providers and their staff to view patient medication use and asthma control status. This is available to providers that enroll their patients with the Propeller Health System but can be integrated into existing EMR systems to allow for data sharing among providers. Merchant et al. evaluated this enhanced Propeller Health System and found a decrease in asthma-related emergency department visits and hospitalizations [33].

The Hailie ${ }^{\mathrm{TM}}$ solution (previously known as SmartInhaler ${ }^{\mathrm{TM}}$ platform) is a system of medication sensors (e.g., SmartTrack ${ }^{\mathrm{TM}}$, SmartTouch ${ }^{\mathrm{TM}}$, SmartTouch AVTM, SmartTurbo ${ }^{\mathrm{TM}}$; http://www.smartinhaler.com/devices/) that wrap around existing inhalers and automatically sends usage data to a patient's smartphone [34]. The sensors record the date and time of actuations, the total number of actuations used, and missed doses. The device pairs with the Hailie ${ }^{\mathrm{TM}}$ App and enables the patient and healthcare professionals to track medication adherence, set daily reminders, and discover insights into their medication usage. The SmartInhaler ${ }^{\mathrm{TM}}$ sensors have been studied extensively and have demonstrated high user acceptability as well as efficacy in increasing medication adherence $[35,36]$. Five randomized controlled trials using the SmartInhaler ${ }^{\mathrm{TM}}$ have been conducted in the UK, Australia, and New Zealand, including a total of 589 adults and children with asthma in a community setting [35-39]. The authors of these studies conclude that SmartInhaler ${ }^{\mathrm{TM}}$ devices were more effective than standard care in improving adherence to asthma medication. Two of the studies showed significant improvement in clinical outcomes (e.g., asthma exacerbations, asthma control, and healthcare utilization) [37, 38].

The Inhaler Compliance Assessment (INCA) device uses time-stamped audio recordings of inhaler use to assess not only inhaler adherence but also inhaler technique (http://www.tueohealth.com/). Specifically, it can assess proper inhaler technique by measuring a failure to prime the inhaler, inadequate inspiratory flow, and dose dumping. This information can be used to determine if medication was not properly delivered due to inhaler technique errors (attempted adherence) or missed/late medication doses. In one study of 184 people with chronic obstructive pulmonary disease, the INCA device was used to assess controller inhaler adherence [40]. The mean rate of attempted adherence was $58.7 \%$, whereas actual adherence (proper timing and technique) was only $23 \%$. Only $7 \%$ of participants had actual adherence above $80 \%$, underscoring the importance of addressing proper inhaler technique. Currently, there is no mobile app for the INCA tool and the data must be downloaded onto a computer for analysis. Additional studies are currently being conducted using an INCA App to assess its ability to improve asthma outcomes. 
Tueo Health, which was acquired by Apple in 2019, developed a passive EMD to help parents monitor nighttime asthma symptoms in children. Using commercial breathing sensors placed under the mattress, the device can passively measure key physiologic parameters during sleep (http://www. tueohealth.com/). An asthma control score is then calculated from the physiologic data and available for the patient to view through a mobile app. The score gives direct indication of their asthma control status based on their provider-issued asthma action plan and recommends evidence-based steps to manage asthma. Such steps include reminders to avoid known triggers, refill a prescription, or make an appointment to see their healthcare provider.

\section{Use of Mobile Apps in Asthma}

As with wearable devices, the use of health apps on phones and tablets has rapidly been increasing [13]. There are a large number of asthma-related apps with well over 500 reported in 2019 [41 •*]. The mobile apps range from providing health education, symptom tracking, environmental alerts, and medication reminders. A review conducted in 2015 selected 209 English asthma-related apps that were available in Google Play and/or Apple Store [42]. The majority (52\%) of the apps evaluated were teaching and training information for patients, with $39 \%$ based on alternative treatments, such as yoga, acupressure, and breathing exercises; $22 \%$ helped users to monitor symptoms and medication adherence; $18 \%$ provided medical reference information for providers; $6 \%$ provided air quality information; and $2 \%$ served as social forums for patients with asthma.

Despite the great number of currently available mobile apps for asthma, few effectiveness evaluations have been conducted. In 2013, Marcano and colleagues conducted a Cochrane review on mobile apps for asthma self-management. Only two randomized controlled trials (RCTs) fit the review criteria and assessed efficacy of the apps on asthma outcomes such as asthma control, asthma quality of life, and healthcare utilization [43]. Both RCTs $(n=408)$ evaluated the effect of a mobile phone-based asthma self-management intervention on asthma control by comparing it with traditional, paper-based asthma self-management. Overall, the results were inconclusive, as the evidence was low quality (GRADE) and attrition bias plus other sources of bias were found. In 2017, another systematic review assessed asthma self-management using mHealth apps [44]. The authors found that mHealth apps improved asthma control in five studies, lung function in two studies, and quality of life in three studies. There was no significant impact on economic outcomes such as hospitalization rates.

While there is preliminary evidence on the efficacy of asthma self-management mobile apps to improve asthma control and quality of life, the mechanisms have not been well studied. Ramsey and colleagues reviewed the content and quality of mHealth asthma self-management apps [45••]. The mobile apps were identified through systematic searches of the Apple and Google Play stores and then coded to evaluate the inclusion of evidence-based behavior change techniques (BCTs). Examples of BCTs include providing information about behavior-health link, identifying barriers to performing health behaviors, and prompting self-monitoring of behaviors. The quality of the apps was also rated using the Mobile App Rating Scale (MARS) to determine app engagement, functionality, aesthetics, information quality, and subjective quality. Of the twenty-three apps identified, a range of 1-11 BCTs were used by the apps. The most commonly included BCTs were instruction, behavior-health link, self-monitoring, feedback, teach to use prompts/cues, consequences, and others' approval. Three apps used at least eight BCTs (i.e., AsthmaMD, Kiss myAsthma, and My Breathefree). The overall quality of the apps, as measured by MARS, ranged from 2.45 to 4.50 (out of a possible 5.00). Four apps had an overall MARS quality of $>4.0$ (AsthmaMD, Asthma Health Storylines, Kiss myAsthma, and Wizdy Pets: Kids' Asthma Game). As it is more common for patients to ask their healthcare providers to help them choose apps, it is important that providers are knowledgeable about which asthma management apps are high quality and include evidence-based components that will be successful for behavior change. To date, Kiss myAsthma and AsthmaMD are the asthma management apps with the highest number of BCTs and quality scores, but this area is constantly evolving.

There are some limitations to consider regarding the use of mobile apps for asthma. The majority of commercially available asthma apps lack validation in clinical settings. Further, the apps are not regulated or approved as medical devices and may provide recommendations to patients that are not evidence-based or inaccurate. Finally, there is a risk of loss of privacy of health information for patients that use these apps. One study showed that only $30 \%$ of the most commonly used mHealth apps had privacy policies [46]. These policies often required a high-level of literacy for comprehension, were often not focused on the app itself, and were not informative for end users. Therefore, clinicians should evaluate asthma management apps to ensure high quality and evidenced-based information prior to recommending them for their asthma patients.

\section{Conclusion: a New Wave of Wearable Devices}

The world and medicine as we know it are changing forever in the wake of the repercussions of the COVD-19 pandemic. Limiting unnecessary in-person visits, especially for at-risk patients, and expanding the scope of what we can provide 
remotely using new technological advancements should be a priority moving forward. The future of wearable devices seems to be headed towards continuous measurements of personal physiology to predict, prevent, or help to aid in the diagnosis of an underlying health condition. This approach allows frequent and continuous measurements of body functions including heart rate, skin temperature, blood oxygen levels, and physical activity. Recent studies investigating the utility of wearable sensors indicate that the information provided by these devices is physiologically meaningful and actionable and will likely to play an important role in managing health in the future [47]. Healthcare providers and patients themselves can do a much better job of tracking their health outside of regularly scheduled office visits. Currently, providers are relying on patients with chronic health conditions to consistently self-report progress and medication compliance which is inefficient and unpredictable at best. Having access to reliable home data on a consistent basis that is relevant to the patient's specific medical condition is key to improving health outcomes not only for asthma but also for all chronic diseases. Increased utilization of health monitoring devices by patients will likely become an important aspect of self-care and preventive medicine moving forward. The data provided by these devices will allow care providers to provide patients with personalized tools and tailored solutions to improve their health and encourage fitness and diet goals. They may also help to enhance accurate symptom reports, improve accuracy of diagnosis, and streamline appropriate specialty referral when appropriate.

\section{Compliance with Ethical Standards}

Conflict of Interest Dr. Greiwe is a consultant for AstraZeneca on social media and respiratory biologics scientific expert on the virtual advisory board; Sanofi Grenzyme Dupixent scientific expert on the virtual advisory board; honoraria from AstraZeneca Fesenra Speakers Bureau, Regeneron, and Sanofi Grenzyme Dupixent Speakers Bureau. Dr. Nyenhuis is a board member of Chicago Asthma Consortium; grant from National Institute of Health, honoraria from Sanofi, royalties from Springer Nature and Wolters Kluwer Health.

Human and Animal Rights and Informed Consent This article does not contain any studies with human or animal subjects performed by any of the authors.

\section{References}

Papers of particular interest, published recently, have been highlighted as:

•• Of major importance

1. Caulfield BM, Donnelly SC. What is connected health and why will it change your practice? QJM. 2013;106(8):703-7. https:// doi.org/10.1093/qjmed/hct114.
2. Tsai AG, Wadden TA. Systematic review: an evaluation of major commercial weight loss programs in the United States. Ann Intern Med. 2005;142(1):56-66.

3. Essery R, Geraghty AW, Kirby S, Yardley L. Predictors of adherence to home-based physical therapies: a systematic review. Disabil Rehabil. 2017;39(6):519-34. https://doi.org/10.3109/09638288. 2016.1153160 .

4. Heshka S, Anderson JW, Atkinson RL, Greenway FL, Hill JO, Phinney SD, et al. Weight loss with self-help compared with a structured commercial program: a randomized trial. JAMA. 2003;289(14):1792-8. https://doi.org/10.1001/jama.289.14.1792.

5. Wing RR, Jeffery RW. Benefits of recruiting participants with friends and increasing social support for weight loss and maintenance. J Consult Clin Psychol. 1999;67(1):132-8.

6. Hwang KO, Ottenbacher AJ, Green AP, Cannon-Diehl MR, Richardson O, Bernstam EV, et al. Social support in an Internet weight loss community. Int J Med Inform. 2010;79(1):5-13. https://doi.org/10.1016/j.ijmedinf.2009.10.003.

7. Lambert TE, Harvey LA, Avdalis C, Chen LW, Jeyalingam S, Pratt $\mathrm{CA}$, et al. An app with remote support achieves better adherence to home exercise programs than paper handouts in people with musculoskeletal conditions: a randomised trial. Aust J Phys. 2017;63(3):161-7.

8. Goto M, Takedani H, Haga N, Kubota M, Ishiyama M, Ito S, et al. Self-monitoring has potential for home exercise programmes in patients with haemophilia. Haemophilia. 2014;20:e121-7.

9. Hutchesson MJ, Rollo ME, Krukowski R, Ells L, Harvey J, Morgan $\mathrm{PJ}$, et al. eHealth interventions for the prevention and treatment of overweight and obesity in adults: a systematic review with metaanalysis. Obes Rev. 2015;16:376-92.

10. Cotter AP, Durant N, Agne AA, Cherrington AL. Internet interventions to support lifestyle modification for diabetes management: a systematic review of the evidence. J Diabetes Complicat. 2014;28: 243-51.

11. Buller DB, Borland R, Bettinghaus EP, Shane JH, Zimmerman DE. Randomized trial of a smartphone mobile application compared to text messaging to support smoking cessation. Telemed J E Health. 2014;20:206-14.

12. Lamkin P. Smart wearables market to double by 2022: $\$ 27$ billion industry forecast. Forbes. 2018. https://www.forbes.com/sites/ paullamkin/2018/10/23/smart-wearables-market-to-double-by2022-27-billion-industry-forecast/\#2d54a9e12656 (accessed 14 Jan 2020).

13. https://www.statista.com/statistics/543070/number-of-wearableusers-in-the-us/ (accessed 3/1/2020).

14. Apple launches three innovative studies today in the new Research app. Apple Newsroom. 2019. https://www.apple.com/newsroom/ 2019/11/apple-launches-three-innovative-studies-today-in-thenew-research-app/ (accessed 14 Jan 2020).

15. Beach MC, Keruly J, Moore RD. Is the quality of the patientprovider relationship associated with better adherence and health outcomes for patients with HIV? J Gen Intern Med. 2006;21:661-5.

16. Bertakis KD, Azari R. Patient-centered care is associated with decreased health care utilization. J Am Board Fam Med. 2011;24: 229-39.

17. Alvarez C, Greene J, Hibbard J, Overton V. The role of primary care providers in patient activation and engagement in self-management: a cross-sectional analysis. BMC Health Serv Res. 2016;16(1):1-8. https://doi.org/10.1186/s12913-016-1328-3.

18.• Castner J, Mammen MJ, Jungquist CR, et al. Validation of fitness tracker for sleep measures in women with asthma. J Asthma. 2019;56:719-30. https://doi.org/10.1080/02770903.2018.1490753 More objective look at fitness trackers' ability to accurately quantify sleep patterns in the home environment as an adjunct to subjective reports. 
19. Koinis-Mitchell D, Kopel SJ, Seifer R, et al. Asthma-related lung function, sleep quality, and sleep duration in urban children. Sleep Health. 2017;3:148-56. https://doi.org/10.1016/j.sleh.2017.03.008.

20. Rosenberger ME, Buman MP, Haskell WL, et al. Twenty-four hours of sleep, sedentary behavior, and physical activity with nine wearable devices. Med Sci Sports Exerc. 2016;48:457-65. https:// doi.org/10.1249/mss.0000000000000778.

21. Bian J, Guo Y, Xie M, Parish AE, Wardlaw I, Brown R, et al. Exploring the association between self-reported asthma impact and Fitbit-derived sleep quality and physical activity measures in adolescents. JMIR mHealth uHealth. 2017;5:e105. Original Paper 25.07.2017. https://doi.org/10.2196/mhealth.7346.

22. Jaimini U, Thirunarayan K, Kalra M, et al. "How is my child's asthma?" Digital Phenotype and Actionable Insights for Pediatric Asthma. JMIR Pediatr Parent. 2018. https://doi.org/10.2196/11988.

23. Venkataramanan R, Thirunarayan K, Jaimini U, et al. Determination of personalized asthma triggers from multimodal sensing and a mobile app: observational study. JMIR Pediatr Parent. 2019;2:e14300. https://doi.org/10.2196/14300.

24. Anusha AR, Soodi AL and Kumar SP. Design of low-cost hardware for lung sound acquisition and determination of inspiratoryexpiratory phase using respiratory waveform. In: 2012 Third International Conference on Computing, Communication and Networking Technologies (ICCCNT'12) 26-28 July 2012 2012, pp.1-5.

25. Liu G-Z, Guo Y-W, Zhu Q-S, Huang BY, Wang L. Estimation of respiration rate from three-dimensional acceleration data based on body sensor network. Telemed J E Health. 2011;17:705-11. https:// doi.org/10.1089/tmj.2011.0022.

26. Boner AL, Piacentini GL, Peroni DG, et al. Children with nocturnal asthma wheeze intermittently during sleep. J Asthma. 2010;47(3): 290-4. 103109/0277090090349718828.

27. Mahler DA. Peak inspiratory flow rate as a criterion for dry powder inhaler use in chronic obstructive pulmonary disease. Ann Am Thorac Soc. 2017;14:1103-7 2017/05/10. https://doi.org/10.1513/ AnnalsATS.201702-156PS.

28. Safioti G, Granovsky L, Li T, et al. A predictive model for clinical asthma exacerbations using albuterol eMDPI (ProAir Digihaler): a 12-week, open-label study. A31 ASTHMA: CLINICAL STUDIES I. Am Thoracic Soc. 2019:A7307-7.

29. https://www.propellerhealth.com/ (accessed 3/5/2020).

30. Van Sickle D, Magzamen S, Truelove S, et al. Remote monitoring of inhaled bronchodilator use and weekly feedback about asthma management: an open-group, short-term pilot study of the impact on asthma control. PLoS ONE. 2013;8:e55335. https://doi.org/10. 1371/journal.pone.0055335.

31.• Merchant RK, Inamdar R, Quade RC. Effectiveness of population health management using the propeller health asthma platform: a randomized clinical trial. J Allergy Clin Immunol Pract. 2016;4(4): 455-63. https://doi.org/10.1016/j.jaip.2015.11.022 Novel look at how adherence tracking using Propeller Health Asthma Platform significantly reduced rescue inhaler use and improved ACT scores.

32. Barrett MA, Humblet O, Marcus JE, et al. Effect of a mobile health, sensor-driven asthma management platform on asthma control. Ann Allergy Asthma Immunol. 2017;119:415-421.e411. https:// doi.org/10.1016/j.anai.2017.08.002.

33. Merchant R, Szefler SJ, Bender BG, et al. Impact of a digital health intervention on asthma resource utilization. World Allergy Org J. 2018;11:28. https://doi.org/10.1186/s40413-018-0209-0.

34. https://www.adherium.com/technology (accessed 3/3/2020).

35. Charles T, Quinn D, Weatherall M, Aldington S, Beasley R, Holt S. An audiovisual reminder function improves adherence with inhaled corticosteroid therapy in asthma. J Allergy Clin Immunol. 2007;119:811-6. https://doi.org/10.1016/j.jaci.2006.11.700.

36. Foster JM, Smith L, Usherwood T, Sawyer SM, Rand CS, Reddel HK. The reliability and patient acceptability of the SmartTrack device: a new electronic monitor and reminder device for metered dose inhalers. J Asthma. 2012;49:657-62. https://doi.org/10.3109/ 02770903.2012.684253.

37. Morton RW, Elphick HE, Rigby AS, Daw WJ, King DA, Smith LJ, et al. STAAR: a randomised controlled trial of electronic adherence monitoring with reminder alarms and feedback to improve clinical outcomes for children with asthma. Thorax. 2017;72:347-54. https://doi.org/10.1136/thoraxjnl-2015-208171.

38. Chan AHY, Stewart AW, Harrison J, et al. The effect of an electronic monitoring device with audiovisual reminder function on adherence to inhaled corticosteroids and school attendance in children with asthma: a randomised controlled trial. Lancet Respir Med. 2015;3:210-9. https://doi.org/10.1016/S2213-2600(15) 00008-9.

39. Burgess SW, Sly PD, Devadason SG. Providing feedback on adherence increases use of preventive medication by asthmatic children. J Asthma. 2010;47:198-201. https://doi.org/10.3109/ 02770900903483840 .

40. Moran C, Doyle F, Sulaiman I, Bennett K, Greene G, Molloy GJ, et al. The INCATM (Inhaler Compliance Assessment ${ }^{\mathrm{TM}}$ ): a comparison with established measures of adherence. Psychol Health. 2017;32:1266-87. https://doi.org/10.1080/08870446.2017. 1290243.

41.• Himes BE, Leszinsky L, Walsh R, et al. Mobile health and inhalerbased monitoring devices for asthma management. J Allergy Clin Immunol Pract. 2019;7:2535-43. https://doi.org/10.1016/j.jaip. 2019.08.034 Nice overview of mobile health and web applications, wearables, and other personal monitoring devices to improve asthma management.

42. Wu AC, Carpenter JF, Himes BE. Mobile health applications for asthma. J Allergy Clin Immunol Pract. 2015;3:446-448.e416. https://doi.org/10.1016/j.jaip.2014.12.011.

43. Marcano Belisario JS, Huckvale K, Greenfield G, et al. Smartphone and tablet self management apps for asthma. Cochrane Database Syst Rev. 2013:Cd010013. 2013/11/28. https://doi.org/10.1002/ 14651858.CD010013.pub2.

44. Farzandipour M, Nabovati E, Sharif R, Arani M, Anvari S. Patient self-management of asthma using mobile health applications: a systematic review of the functionalities and effects. Appl Clin Inform. 2017;08:1068-81. https://doi.org/10.4338/ACI-2017-07-R-0116.

45.• Ramsey RR, Caromody JK, Voorhees SE, et al. A systematic evaluation of asthma management apps examining behavior change techniques. J Allergy Clin Immunol Pract. 2019;7:258391.https://doi.org/10.1016/j.jaip.2019.03.041 Comprehensive evidence-based evaluation of asthma management apps utilizing the presence or absence of behavior change techniques to evaluate efficacy.

46. Sunyaev A, Dehling T, Taylor PL, et al. Availability and quality of mobile health app privacy policies. J Am Med Inform Assoc. 2015;22:e28-33. 2014/08/26. https://doi.org/10.1136/amiajnl2013-002605.

47. Li X, Dunn J, Salins D, et al. Digital health: tracking physiomes and activity using wearable biosensors reveals useful health-related information. PLoS Biol. 2017;15(1):e2001402. Published 2017 Jan 12. https://doi.org/10.1371/journal.pbio.2001402.

Publisher's Note Springer Nature remains neutral with regard to jurisdictional claims in published maps and institutional affiliations. 